Article

\title{
Single-Cell Electric Lysis on an Electroosmotic-Driven Microfluidic Chip with Arrays of Microwells
}

\author{
Chun-Ping Jen ${ }^{1, *}$, Tamara G. Amstislavskaya ${ }^{2}$, Ya-Hui Liu ${ }^{1}$, Ju-Hsiu Hsiao ${ }^{1}$ and \\ Yu-Hung Chen ${ }^{3, *}$
}

1 Department of Mechanical Engineering, Advanced Institute of Manufacturing with High-Tech Innovation, National Chung Cheng University, Chia Yi, 62102, Taiwan;

E-Mails: black_12_28@hotmail.com (Y.-H.L.); ggina03@yahoo.com.tw (J.-H.H.)

2 Institute of Cytology and Genetics, Siberian Branch of Russian Academy of Sciences, Novosibirsk, 630090, Russia; E-Mail: amst@bionet.nsc.ru

3 Department of Biochemistry and Molecular Biology, National Cheng-Kung University, Tainan, 70101, Taiwan

* Authors to whom correspondence should be addressed; E-Mails: imecpj@ccu.edu.tw (C.-P.J.); second@mail.ncku.edu.tw (Y.-H.C.); Tel.: +886-5-272-0411 (ext. 33322); Fax: +886-5-272-0589.

Received: 16 April 2012; in revised form: 15 May 2012 / Accepted: 21 May 2012 /

Published: 25 May 2012

\begin{abstract}
Accurate analysis at the single-cell level has become a highly attractive tool for investigating cellular content. An electroosmotic-driven microfluidic chip with arrays of $30-\mu \mathrm{m}$-diameter microwells was developed for single-cell electric lysis in the present study. The cellular occupancy in the microwells when the applied voltage was $5 \mathrm{~V}(82.4 \%)$ was slightly higher than that at an applied voltage of $10 \mathrm{~V}(81.8 \%)$. When the applied voltage was increased to $15 \mathrm{~V}$, the cellular occupancy in the microwells dropped to $64.3 \%$. More than $50 \%$ of the occupied microwells contain individual cells. The results of electric lysis experiments at the single-cell level indicate that the cells were gradually lysed as the DC voltage of $30 \mathrm{~V}$ was applied; the cell was fully lysed after $25 \mathrm{~s}$. Single-cell electric lysis was demonstrated in the proposed microfluidic chip, which is suitable for high-throughput cell lysis.
\end{abstract}

Keywords: microwell; single-cell; electric lysis; electroosmotic-driven; microfluidics 


\section{Introduction}

Conventional biochemical assays are performed using populations of cells to determine their quantitative biomolecular profiles. However, population averages do not reflect actual physiological processes in individual cells, which occur either on very short time scales (e.g., kinase signaling cascades) or nonsynchronously (e.g., response to an external chemical gradient) [1]. Biomedical devices created using microfabrication technologies allow the precise manipulation of biological cells, and thus have the potential to provide individual characterization, detection, and assay of cells at the single-cell level. Therefore, accurate analysis at the single-cell level has become a highly attractive tool for investigating cellular content. Microfabrication technologies combined with surface chemistry have stimulated research to understand the fundamental cell biology and pharmaceutical analysis by exposure of cells to drugs and environmental perturbations [2]. Many methods, such as microcontact printing, microfluidic patterning, and photolithography, have been employed to create micropatterned surfaces containing adhesive and non-adhesive regions for cells [3-6]. These approaches are limited to adherent cells and additional surface chemistry procedures are often required. Alternative methods that do not require adherent cells, including dielectrophoresis [7], optical tweezers [8] and selective dewetting [9], have been adopted for trapping single cells. However, these methods are not suitable for high-throughput applications. The approach of passively confining cells inside microwells has been proposed because of its simplicity and ease of operation. Park et al. [10] proposed a flow method that enables single-cell trapping in microwells with a size sufficient to allow attachment and division of captured cells. A simple method for trapping single cells in large open-top microwell arrays was developed and optimized by Rettig and Folch [11]. The parameters that maximize single-cell occupancy for two cell types, including the microwell diameter, microwell depth, and settling time, were also investigated in their study. Cell lysis is crucial in the analysis of intracellular components containing information about genetic or disease characteristics in genomics, proteomics, and metabolomics [12]. Cell lysis could be achieved by various approaches [13], such as optical [14], mechanical [15], chemical [16], and electric [17] methods. A method that combines optical trapping and microfluidic-based droplet generation for encapsulating single cells within a picolitre-size aqueous droplet was proposed by $\mathrm{He}$ et al. [18]. The trapped cells are lysed rapidly using a YAG laser with a 5-ns pulse duration. Continuous analysis of two dyes loaded into single mammalian cells using laser-based lysis combined with the electrophoretic separation of cell content was achieved using microfluidic chips [19]. The cells are mechanically lysed owing to a cavitation bubble generated by a single laser pulse from a 532-nm picosecond pulsed laser. Laser-mediated lysis is well suited for integration into microfluidic chip platforms; however, it requires complex experimental setups. In chemical lysis, a detergent is usually introduced into a cell membrane to create pores within the membrane and lyse the cell. Detergent lysis is well established for bulk biochemical assays. Single-cell capture by a dam-like structure and chemical lysis inside a closed volume was demonstrated in a microfabricated device [16]. Following cell lysis, a limited and stable dilution of intracellular components is used to simplify the requirements for downstream assays. Huang et al. [20] designed a microfluidic device to trap cells using a pair of valves. Then, the chamber, where the cell is immobilized, is filled with lysis buffer containing fluorescent antibodies for labeling proteins to quantify the protein contents of a single cell using single-molecule fluorescence counting. Adherent 
cells were analyzed serially using detergent lysis followed by capillary electrophoresis [21]. Electrophoretic buffer containing sodium dodecyl sulfate (SDS), which is a strong ionic detergent that achieves cell lysis on the order of seconds, was then introduced using sheath flow around the capillary inlet. A simple single-cell lysis method that uses a dense array of microwells (10-30 pL in volume) fabricated from poly(dimethylsiloxane) (PDMS) and a commercially available cell lysis reagent was developed [22]. After the cell lysis solution diffuses into a microwell from the flow cell, which is between a bottom coverslip and a top cell-trapping PDMS sheet separated by two strips of double-faced adhesive tape, the PDMS sheet is rapidly pressed against the bottom coverslip to close each microwell, thereby causing gradual cell lysis. McClain et al. [17] developed an integrated microfluidic device that automatically transports cells to an electrical lysis location. The cell lysate is injected into a separation channel and the labeled lysate contents are electrophoretically separated prior to laser-induced fluorescence (LIF) detection. A microfluidic device with different field strengths in geometrically defined sections of a microchannel was proposed to analyze intracellular contents from single cells after electrical lysis [23]. The electrophoresis of calcein AM (a fluorogenic dye) which was loaded in the cytoplasm prior to electric lysis was demonstrated the feasibility of analyzing cell lysate from single cells in their device. Furthermore, microfluidic devices with different geometries providing pulse-like electric field variation were applied to gene delivery by electroporation [24]. A continuous electrical cell lysis device in which the width and length of the microchannel changes to generate a focused high electric field strength for cell lysis was proposed [25]. A low electric field strength for the transport of samples at a low operational voltage is employed. The device generates a high electric field strength of $1.2 \mathrm{kV} / \mathrm{cm}$ at the orifice to disrupt red blood cells with a $100 \%$ lysis rate under an operational voltage of $50 \mathrm{~V}$. A device capable of electrical cell lysis and the evaluation of lysis efficiency in continuous flow using dielectrophoretic cell sorting was proposed by Mernier et al. [26]. An AC electrical field is used at a frequency that optimizes cell lysis while avoiding the creation of bubbles at the electrode surface; the AC field causes a dielectrophoretic effect on the cells that can be used to increase the transit time of the cell in the lysis region. In the present study, a microfluidic chip with microwells of $30 \mu \mathrm{m}$ in diameter, is developed for cellular patterning using a electroosmoticdriven flow and the feasibility of electric lysis for human carcinoma cells (HeLa cells) at the single-cell level is demonstrated.

\section{Materials and Methods}

\subsection{Fabrication of Microfluidic Chips with Microwells}

A biocompatible material, poly(dimethylsiloxane) (PDMS), was adopted for single-cell-based arrays in the microfluidic chip, as illustrated in Figure 1(a). The main channel, formed on the top PDMS layer, is $4.4 \mathrm{~mm}$ wide, $100 \mu \mathrm{m}$ in height and $21 \mathrm{~mm}$ long. This main channel is divided into three microchannels, each $800 \mu \mathrm{m}$ wide and $8 \mathrm{~mm}$ long, at the center region. Each microchannel contains four $10 \times 10$ microwells with $30 \mu \mathrm{m}$ in diameter and $20 \mu \mathrm{m}$ deep, on the bottom PDMS layer. The mold masters were fabricated by spinning SU-8 (SU-8 50, MicroChem Corp., Newton, MA, USA) on a silicon wafer to define the microwells and microchannel, respectively. The mold master of the microfluidic channels (around $100 \mu \mathrm{m}$ in height) was fabricated by spinning SU-8 at $500 \mathrm{rpm}$ for 
$20 \mathrm{~s}$ and then at $1,000 \mathrm{rpm}$ for $35 \mathrm{~s}$ on the silicon wafer. The resist was soft baked on a hotplate at $65{ }^{\circ} \mathrm{C}$ for $10 \mathrm{~min}$ and then at $95{ }^{\circ} \mathrm{C}$ for $30 \mathrm{~min}$. The resist was then allowed to cool to room temperature. The SU-8 was exposed to ultraviolet (UV) radiation at a dose of $200 \mathrm{~mJ} / \mathrm{cm}^{2}$. The post-exposure baking was done at $65^{\circ} \mathrm{C}$ for $3 \mathrm{~min}$ and then at $95{ }^{\circ} \mathrm{C}$ for $10 \mathrm{~min}$. The exposed samples were developed with SU-8 developer for $5 \mathrm{~min}$. The mold master of the microwells (around $20 \mu \mathrm{m}$ in height) was fabricated by spinning SU-8 at $500 \mathrm{rpm}$ for $20 \mathrm{~s}$ and then at $4,500 \mathrm{rpm}$ for $35 \mathrm{~s}$ on a silicon wafer. The resist was developed with SU-8 developer for about 2 min after baking and exposure to UV radiation under the conditions mentioned above. PDMS prepolymer mixture (Sylgard-184 Silicone Elastomer Kit, Dow Corning, Midland, MI, USA) was poured and cured on the mold masters to replicate the patterned structures. After peeling off the PDMS replica with the microchannel, the inlet and outlet ports were made by a puncher. The two PDMS replicas were bonded after treatment with oxygen plasma in an $\mathrm{O}_{2}$ plasma cleaner (model PDC-32G, Harrick Plasma Corp., Ithaca, NY, USA). Electrodes were inserted into the inlet and outlet for applying the voltages. The distance between two electrodes is $15 \mathrm{~mm}$. A photograph of the completed microfluidic chip is shown in Figure 1(b).

Figure 1. (a) Schematic diagram of the proposed microfluidic chip for single-cell-based microarrays; (b) Photograph of the completed microfluidic chip.

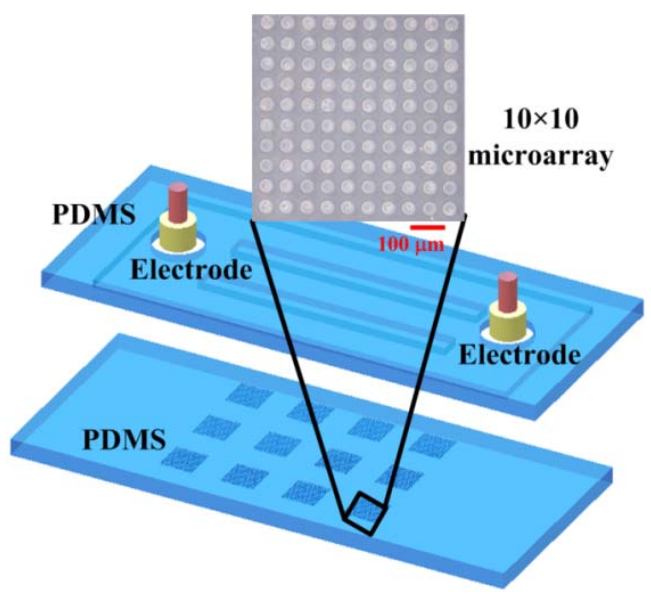

(a)

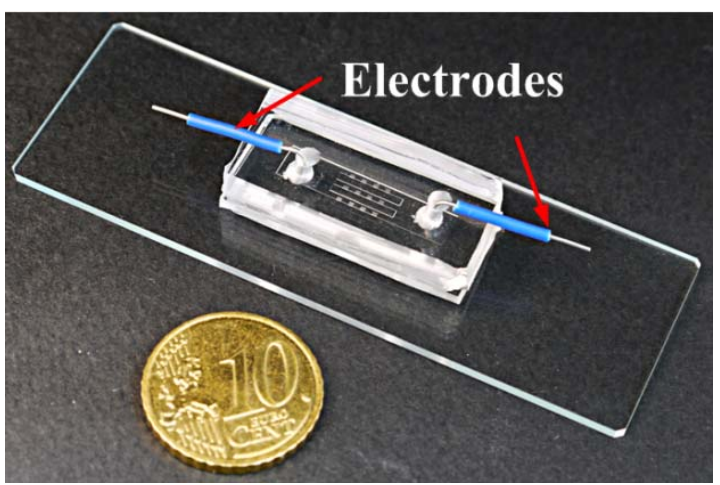

(b)

\subsection{Cell Treatment}

A human cervical carcinoma cell line (HeLa cells) was cultured for an experimental demonstration of single-cell lysis using the proposed microfluidic chips with microwells. The cells were serially passaged as monolayer cultures in DMEM medium (Gibco, Grand Island, NY, USA), with $3.7 \mathrm{~g}$ of $\mathrm{NaHCO}_{3}$ per liter of medium added, supplemented with $10 \%$ fetal bovine serum (FBS, Gibco) and $1 \%$ penicillin/streptomycin (Gibco). The cell culture dish (Falcon, Franklin Lakes, NJ, USA) was incubated in a humidified atmosphere containing $5 \%$ carbon dioxide at $37{ }^{\circ} \mathrm{C}$; the medium was replaced every 1 to 2 days. Cells grown to sub-confluence were washed with phosphate-buffered saline (PBS, Biochrome, $\mathrm{pH} 7.4$ ) and harvested by a 5 -minute treatment with $0.25 \%$ trypsin and $0.02 \%$ ethylene diamine tetraacetic acid (EDTA, Sigma, St. Louis, MO, USA). The cells were stained using a standard fluorescence assay with calcein AM (Molecular Probes, Eugene, OR, USA) prior to the experiment. Calcein AM is a green fluorescent dye which is able to penetrate the cell membrane into 
the cytosol and transform into a fluorescent form when it is hydrolyzed by esterases located inside cells. The cell samples were then suspended in an $8.62 \mathrm{wt} \%$ sucrose solution to increase the osmolarity to normal physiological levels.

\subsection{Experimental Procedure}

The experimental procedures are illustrated in Figure 2. First of all, the microfluidic channel was washed and blocked overnight with bovine serum albumin (BSA) buffer $(10 \mathrm{mg} / \mathrm{mL})$ to avoid nonspecific absorption of cells. The sucrose solution was injected manually to replace the blocking buffer prior to experiments. The trapped bubbles within the microwells were removed using an ultrasonic vibrator. The cell sample of $6 \mu \mathrm{L}$, which has a concentration of $10^{7}$ cells $/ \mathrm{mL}$ was dropped, and then a direct current (DC) voltage of 5, 10 and $15 \mathrm{~V}$ was applied for 45 min to electroosmotically drive the cells into the microwells. The sucrose solution was used herein as a working fluid for electroosmosis to avoid the occurrences of joule heating and bubble formation due to the medium with a high conductivity, for example the phosphate saline buffer (PBS) [27]. The microfluidic channel was washed with PBS buffer after that the chip was placed still for 10 min. Finally, a DC voltage of $30 \mathrm{~V}$ was applied to perform the electric lysis in the PBS buffer with a high conductivity, in which the required voltage for lysis could be reduced. The cellular deposition in microwells and the electric lysis were then observed and recorded by an inverted fluorescence microscope (model CKX41, Olympus, Tokyo, Japan) mounted on a CCD camera (DP71, Olympus, Tokyo, Japan) and controlled by a computer with Olympus DP controller image software. The fluorescent images were quantitatively analyzed using NIH ImageJ (National Institute of Health, Bethesda, MD, USA) to measure the intensity of fluorescence. The fluorescence intensity from each pixel can be analyzed with the ImageJ program.

Figure 2. Experimental procedures for cell patterning.

1. Fill the microchannels with BSA buffer and cooled to $10^{\circ} \mathrm{C}$ overnight

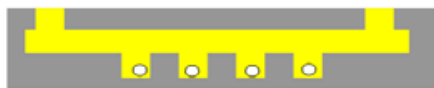

2. Replace BSA buffer with sucrose buffer

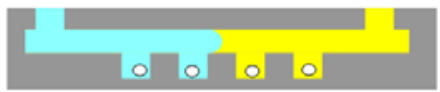

3. Remove the bubbles by using an ultrasonic vibrator

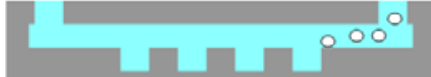

4. Introduce HeLa cells of $6 \mu \mathrm{L}$ and apply DC voltage for $\mathbf{4 5}$ minutes

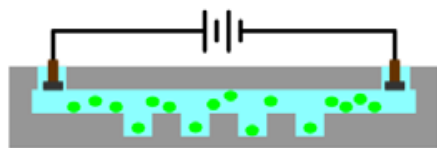

5. Place the chip still for 10 minutes

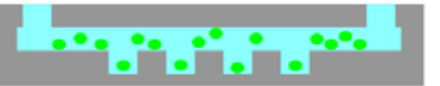

6. Replace sucrose buffer with PBS and washed out the unpatterned HeLa cells

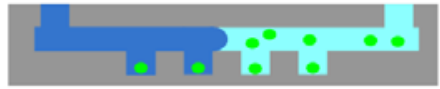

7. Apply 30-volts DC voltage to lysis HeLa cells

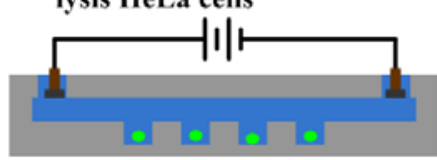

I. Electrode

Bubble

HeLa cell
PBS buffer

Sucrose buffer

BSA

(Bovine Serum Albumin)

PDMS 


\section{Results and Discussion}

Micropatterned HeLa cells in the microfluidic chips with $30-\mu$ m-diameter microwells are shown in Figure 3; the applied voltages for electroosmotic flow are 5, 10 and $15 \mathrm{~V}$, respectively. The occupancy of cells in the microwells when the voltages of 5 or $10 \mathrm{~V}$ are applied is higher than that for the applied voltage is $15 \mathrm{~V}$. The fluorescent images of cells stained by calcein AM demonstrate the viability of the cells.

Figure 3. Bright and fluorescence images of micropatterned HeLa cells in microwells with diameters of $30 \mu \mathrm{m}$ under different applied voltage for electroosmotic flow.

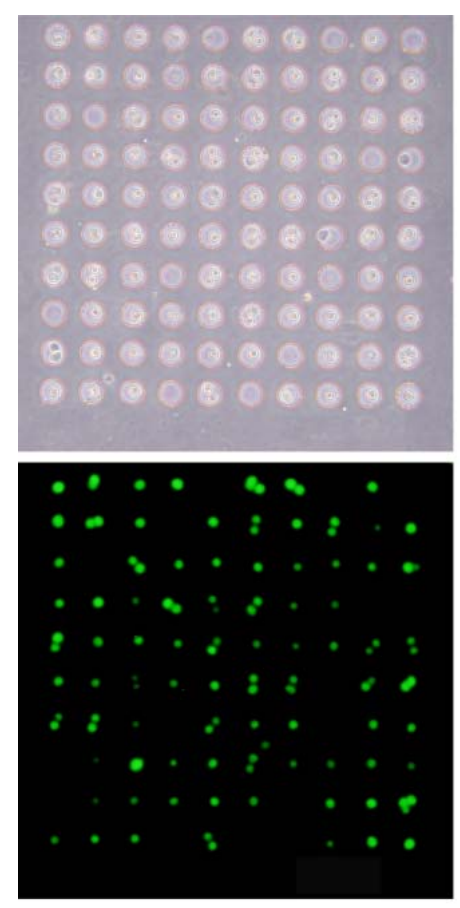

$5 \mathrm{~V}$
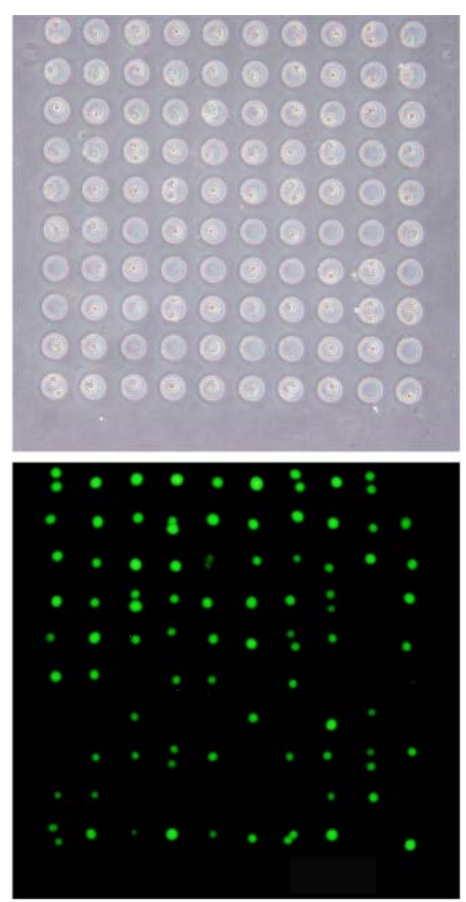

$10 \mathrm{~V}$
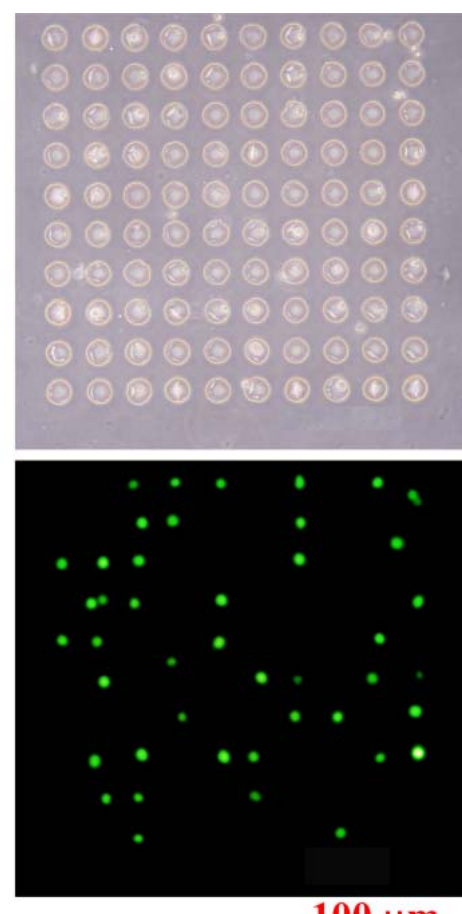

$100 \mu \mathrm{m}$

The electroosmotic-driven velocity of HeLa cells for different applied voltages are measured and plotted in Figure 4. The time of HeLa cells migrating a fixed distance $(50 \mu \mathrm{m})$ was measured via the microscope. The measurements were repeated for more than three times for various applied voltages. The velocity of HeLa cells increased with the applied voltages, as shown in Figure 4. The experimental data of cell occupancy for HeLa cells in the microwells for various applied voltages are revealed in Figure 5. The experimental data is based on manual counts of cells in twelve arrays of $10 \times 10$ microwells using the inverted fluorescence microscope. Each experimental data point represents the average value, and the error bar shows the standard error of the mean. The occupancy of cells in the microwells decreases with increasing applied voltage due to the increasing velocity of HeLa cells. The cellular occupancy in the microwells when the applied voltage was $5 \mathrm{~V}(82.4 \%)$ was slightly higher than that in that $(81.8 \%)$ at applied voltage of $10 \mathrm{~V}$. When the applied voltage was increased to $15 \mathrm{~V}$, the cellular occupancy in the microwells was dropped to $64.3 \%$. More than $50 \%$ of the occupied microwells contain individual cells, as shown in Figure 5. Some of the $30-\mu \mathrm{m}$-diameter microwells contain two or three cells. 
Figure 4. The electroosmotic-driven velocity of HeLa cells for different applied voltages.

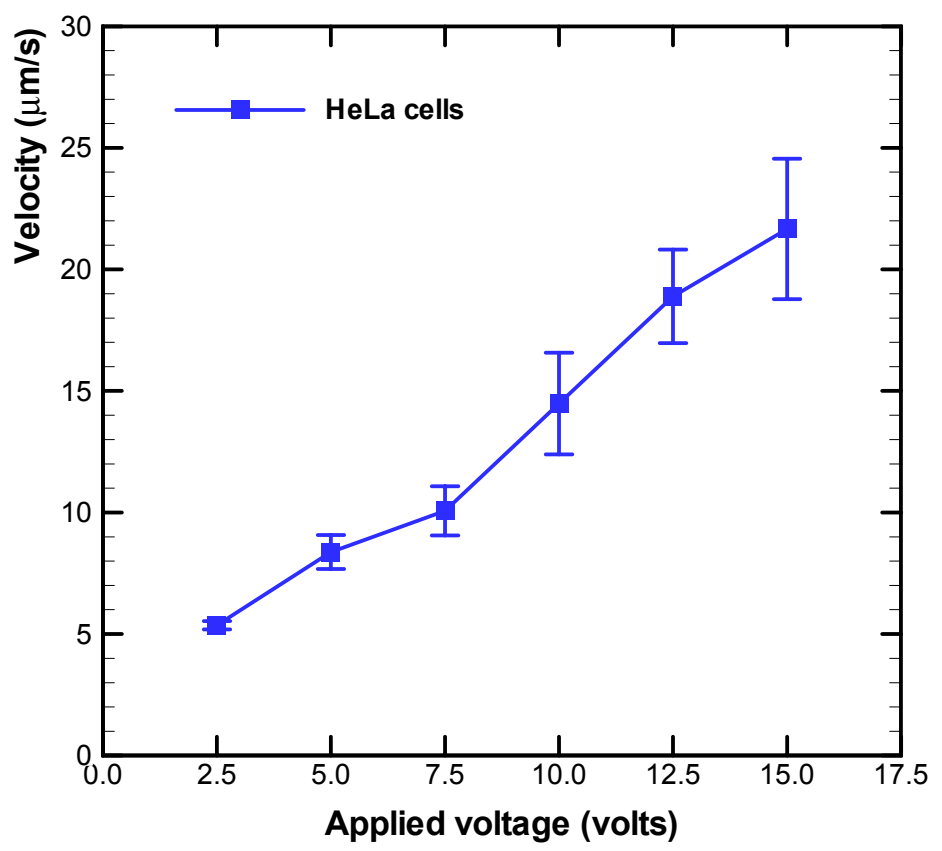

Figure 5. Distributions of 30- $\mu$ m-diameter microwell occupancies for HeLa cells under different applied voltage for electroosmotic flow. The experimental data are based on manual counts of cells in twelve arrays of $10 \times 10$ microwells. Each experimental data point represents the average value and the error bar shows the standard error of the mean.

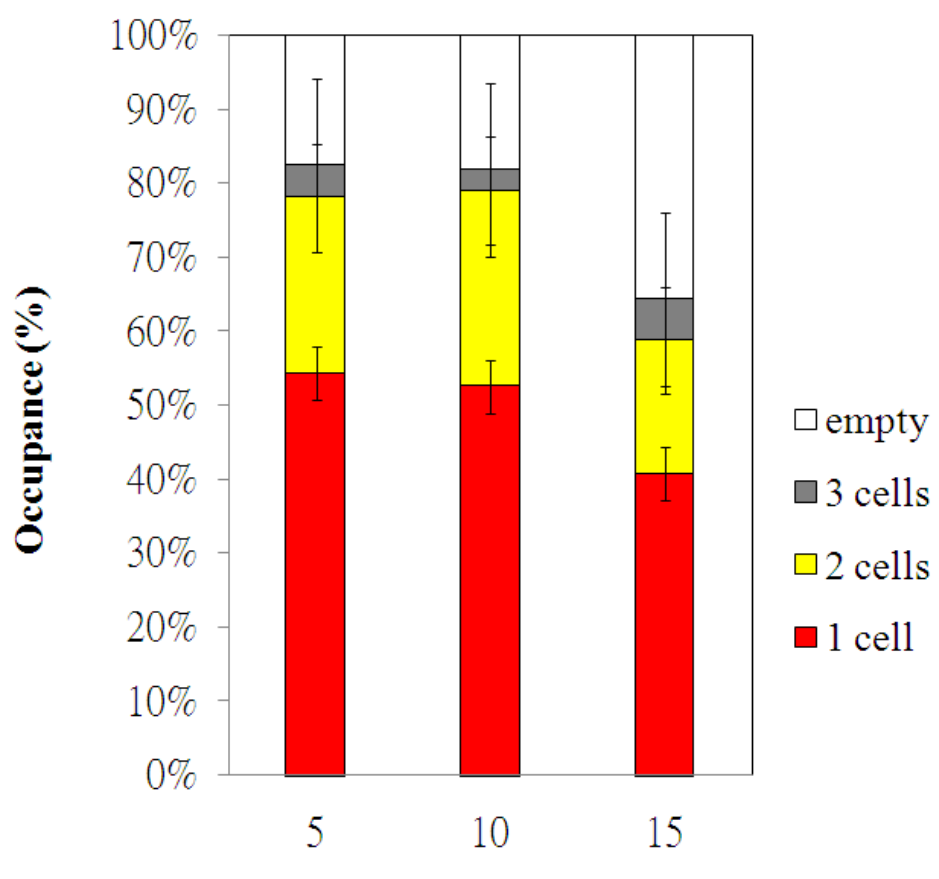

\section{Applied voltage (volts)}

Images showing fluorescence intensity of calcein AM-loaded HeLa cells after applying the voltage of $30 \mathrm{~V}$ for electric lysis are shown in Figure 6(a). The averaged electric field, which is the applied voltage divided by the distance between the electrodes, was about $2 \mathrm{kV} / \mathrm{m}$ and assumed to be unaffected by the presence of cells. The fluorescence images indicate that the cell membranes were 
gradually lysed as the voltage for electric lysis was applied. Calcein leakage occurs when cell membranes are damaged; therefore, the fluorescence intensity within the cell decreases after the cell starts to lyse. The cell was fully lysed after $25 \mathrm{~s}$, as shown in Figure 6(a). The bright-field images of the cell before and after lysis are shown in Figure 6(b). The cell membrane was damaged by the electric field and the cell swelled. However, the cells remained in the microwells after lysis.

Figure 6. (a) Fluorescence images after applying the voltage of $30 \mathrm{~V}$ for electric lysis; (b) Bright-field images of a single HeLa cell before and after lysis.

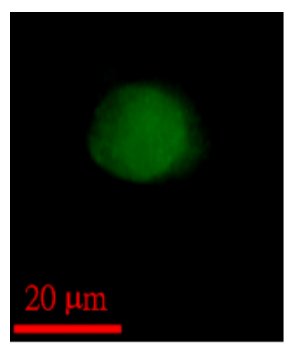

$\mathbf{0} \mathbf{s}$

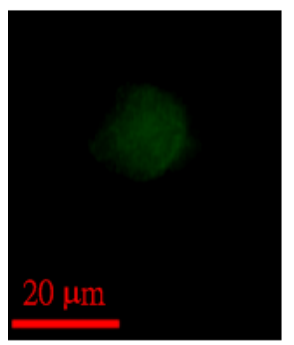

$15 \mathrm{~s}$

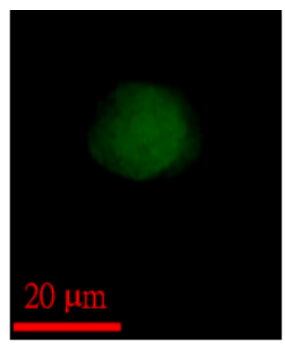

$5 \mathrm{~s}$

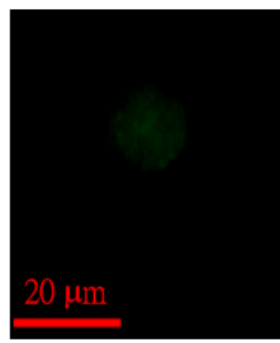

$20 \mathrm{~s}$

(a)

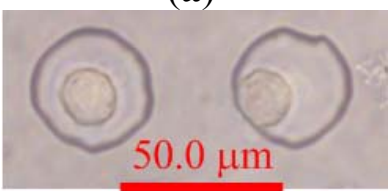

Before lysis

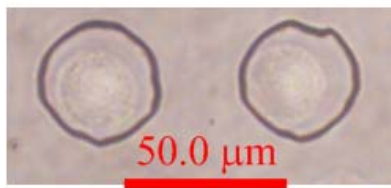

After lysis

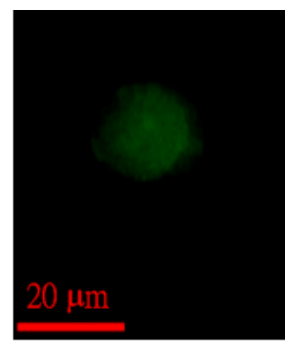

$10 \mathrm{~s}$

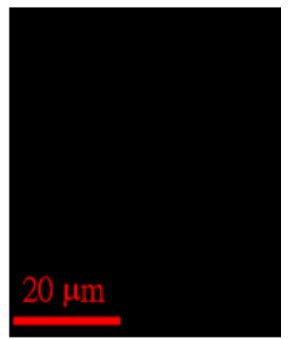

$25 \mathrm{~s}$

(b)

The fluorescence intensities within single cells were quantified using ImageJ software. The fluorescence intensity of cells versus time is plotted in Figure 7. The experimental data are based on measurements of calcein intensity in at least three individual cells. Each experimental data point represents the average value, and the error bar shows the standard error of the mean. The intensity of calcein drops gradually during a DC voltage of $30 \mathrm{~V}$ for electric lysis was applied. The fluorescence intensity decreases to almost zero at $25 \mathrm{~s}$ after applying the voltage. Single-cell-based electric lysis in an electroosmotic-driven microfluidic device with microwells is thus feasible. The present approach of passively confining cells inside microwells is simple to implement and easy to integration. As a cell 
flows through a microchannel, it will gradually settle toward the bottom surface due to gravity and follow streamlines leading into the microwells while losing velocity. Therefore, the issue of stress-activated signaling pathways of cells in the microchips of the flow-based single cell positioning [28,29] could be avoided. The aforementioned approaches of patterning single cells in microwells for single cell analysis [15,22] required manual handling which was not reliable. The electroosmotic-driven microfluidic chip with microwells created by soft lithography are developed in this study, making it low-cost and easy to fabricate.

Figure 7. Fluorescence intensity of a single HeLa cell versus time after applying the voltage of $30 \mathrm{~V}$ for electric lysis. The experimental data are based on measurements of fluorescence in at least three individual cells. Each experimental data point represents the average value and the error bar shows the standard error of the mean.

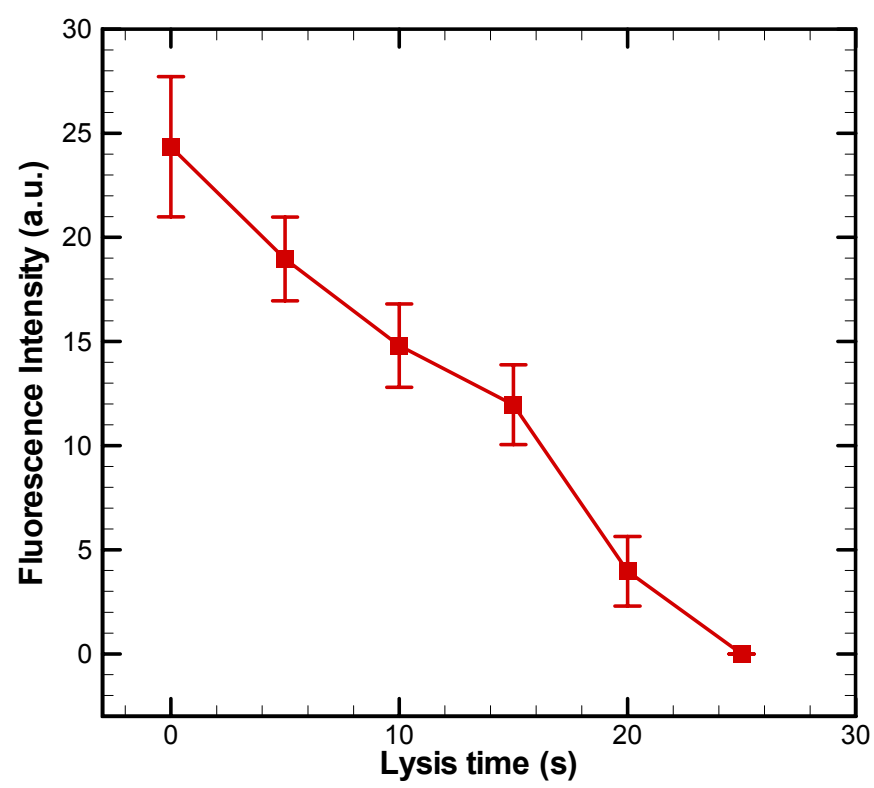

\section{Conclusions}

An electroosmotic-drive microfluidic chip with microwells were fabricated and investigated in the present work. The occupancy of cells in the $30-\mu$ m-diameter microwells was higher than $80 \%$ when the voltages of 5 and $10 \mathrm{~V}$ were applied. The occupancy of cells decreased with increasing the applied voltage. More than $50 \%$ of the occupied microwells contained individual cells. The results of electric lysis experiments at the single-cell level indicate that the cells were gradually lysed as the DC voltage of $30 \mathrm{~V}$ was applied; the cell was fully lysed after $25 \mathrm{~s}$. The bright-field images of the cell before and after lysis indicate that the cell was lysed by the electric field. However, some cell lysate might dissipate in our microchips. The procedures of encapsulating cell lysate inside the microwells, for example, pressing the top PDMS sheet against the bottom one by microfluidic valves, should be investigated in the future. Moreover, the cell viability could be analyzed and remained during electroosmosis. The proposed microfluidic chips are suitable for high-throughput cell lysis and subsequent single-cell analysis, such as monitoring protein levels and enzymatic activities in a single cell, or single-cell PCR. 


\section{Acknowledgment}

The authors would like to thank the National Science Council of Taiwan for financial support under grant NSC 99-2923-E-194-001-MY3 and NSC 100-2113-M-006-007-MY2.

\section{References}

1. Price, A.K.; Culbertson, C.T. Chemical analysis of single mammalian cells with microfluidics. Anal. Chem. 2007, 79, 2614-2621.

2. Yoshimoto, K.; Ichinoa, M.; Nagasaki, Y. Inverted pattern formation of cell microarrays on poly(ethylene glycol) (PEG) gel patterned surface and construction of hepatocyte spheroids on unmodified PEG gel microdomains. Lab. Chip. 2009, 9, 1286-1289.

3. Folch, A.; Toner, M. Microenginggering of cellular interactions. Annu. Rev. Biomed. Eng. 2000, 2, 227-256.

4. Park, T.H.; Shuler, M.L. Integration of cell culture and microfabrication technology. Biotechnol. Prog. 2003, 19, 243-253.

5. Falconnet, D.; Csucs, G.; Grandin, H.M.; Textor, M. Surface engineering approaches to micropattern surfaces for cell-based assays. Biomaterials 2006, 27, 3044-3063.

6. Lim, J.Y.; Donahue, H.J. Cell sensing and response to micro- and nanostructured surfaces produced by chemical and topographic patterning. Tissue Eng. 2007, 13, 1879-1891.

7. Voldman, J.; Gray, M.L.; Toner, M.; Schmidt, M.A. A microfabrication-based dynamic array cytometer. Anal. Chem. 2002, 74, 3984-3990.

8. Ashkin, A. Optical trapping and manipulation of neutral particles using lasers. Proc. Natl. Acad. Sci. USA 1997, 94, 4853-4860.

9. Klauke, N.; Smith, G.L.; Cooper, J.M. Stimulation of single isolated adult ventricular myocytes within a low volume using a planar microelectrode array. Biophys. J. 2003, 85, 1766-1774.

10. Park, J.Y.; Morgan, M.; Sachs, A.N.; Samorezov, J.; Teller, R.; Shen, Y.; Pienta, K.J.; Takayama, S. Single cell trapping in larger microwells capable of supporting cell spreading and proliferation. Microfluid. Nanofluid. 2010, 8, 263-268.

11. Gromov, P.; Celis, J.E.; Gromova, I.; Rank, F.; Timmermans-Wielenga, V.; Moreira, J.M. A single lysis solution for the analysis of tissue samples by different proteomic technologies. Mol. Oncol. 2008, 2, 368-379.

12. Rettig, J.R.; Folch, A. Large-scale single-cell trapping and imaging using microwell arrays. Anal. Chem. 2005, 77, 5628-5634.

13. Brown, R.B.; Audet, J. Current techniques for single-cell lysis. J. R. Soc. Interface 2008, 5, S131-S138.

14. Rau, K.R.; Guerra, A.; Vogel, A.; Venugopalan, V. Investigation of laser-induced cell lysis using time-resolved imaging. Appl. Phys. Lett. 2004, 84, 2940-2942.

15. Di Carlo, D.; Jeong, K.H.; Lee, L.P. Reagentless mechanical cell lysis by nanoscale barbs in microchannels for sample preparation. Lab. Chip. 2003, 3, 287-291.

16. Irimia, D.; Tompkins, R.G.; Toner, M. Single-cell chemical lysis in picoliter-scale closed volumes using a microfabricated device. Anal. Chem. 2004, 76, 6137-6143. 
17. McClain, M.A.; Culbertson, C.T.; Jacobson, S.C.; Allbritton, N.L.; Sims, C.E.; Ramsey, J.M. Microfluidic devices for the high-throughput chemical analysis of cells. Anal. Chem. 2003, 75, 5646-5655.

18. He, M.; Edgar, J.S.; Jeffries, G.D.; Lorenz, R.M.; Shelby, J.P.; Chiu, D.T. Selective encapsulation of single cells and subcellular organelles into picoliter- and femtoliter-volume droplets. Anal. Chem. 2005, 77, 1539-1544.

19. Phillips, K.S.; Lai, H.H.; Johnson, E.; Sims, C.E.; Allbritton, N.L. Continuous analysis of dye-loaded, single cells on a microfluidic chip. Lab. Chip. 2011, 11, 1333-1341.

20. Huang, B.; Wu, H.; Bhaya, D.; Grossman, A.; Granier, S.; Kobilka, B.K.; Zare, R.N. Counting low-copy number proteins in a single cell. Science 2007, 315, 81-84.

21. Marc, P.J.; Sims, C.E.; Allbritton, N.L. Coaxial flow system for chemical cytometry. Anal. Chem. 2007, 79, 9054-9059.

22. Sasuga, Y.; Iwasawa, T.; Terada, K.; Oe, Y.; Sorimachi, H.; Ohara, O.; Harada, Y. Single-cell chemical lysis method for analyses of intracellular molecules using an array of picoliter-scale microwells. Anal. Chem. 2008, 80, 9141-9149.

23. Wang, H.-Y.; Lu, C. Microfluidic chemical cytometry based on modulation of local field strength. Chem. Commun. 2006, 33, 3528-3530.

24. Wang, H.-Y.; Lu, C. Microfluidic electroporation for delivery of small molecules and genes into cells using a common DC power supply. Biotechnol. Bioeng. 2008, 100, 579-586.

25. Lee, D.W.; Cho, Y.H. A continuous electrical cell lysis device using a low dc voltage for a cell transport and rupture. Sens. Actuators B 2007, 124, 84-89.

26. Mernier, G.; Piacentini, N.; Braschler, T.; Demierre, N.; Renaud, P. Continuous-flow electrical lysis device with integrated control by dielectrophoretic cell sorting. Lab. Chip. 2010, 10, 2077-2082.

27. Shafiee, H.; Caldwell, J.L.; Sano, M.B.; Davalos, R.V. Contactless dielectrophoresis: A new technique for cell manipulation. Biomed. Microd. 2009, 11, 997-1006.

28. van den Brink, F.T.G.; Gool, E.; Frimat, J.P.; Bomer, J.; van den Berg, A.; Le Gac, S. Parallel single-cell analysis microfluidic platform. Electrophoresis 2011, 32, 3094-3100.

29. Chung, K.; Rivet, C.A.; Kemp, M.L.; Lu, H. Imaging single-cell signaling dynamics with a deterministic high-density single-cell trap array. Anal. Chem. 2011, 83, 7044-7052.

(C) 2012 by the authors; licensee MDPI, Basel, Switzerland. This article is an open access article distributed under the terms and conditions of the Creative Commons Attribution license (http://creativecommons.org/licenses/by/3.0/). 